\title{
Convolution-Based Truncation Correction for C-Arm CT using Scattered Radiation
}

\author{
Bastian Bier ${ }^{1}$, Chris Schwemmer ${ }^{1,2}$, Andreas Maier ${ }^{1,3}$, Hannes G. Hofmann ${ }^{1}$, \\ Yan $\mathrm{Xia}^{1}$, Joachim Hornegger ${ }^{1,2}$, Tobias Struffert ${ }^{4}$ \\ ${ }^{1}$ Pattern Recognition Lab, Universität Erlangen-Nürnberg \\ 2 Erlangen Graduate School in Advanced Optical Technologies (SAOT) \\ ${ }^{3}$ Siemens AG, Healthcare Sector, Forchheim, Germany \\ ${ }^{4}$ Department of Neuroradiology, Universitätsklinikum Erlangen \\ bastian.bier@medtech.stud.uni-erlangen.de
}

\begin{abstract}
Patient dose reduction in C-arm computed tomography by volume-of-interest (VOI) imaging is becoming an interesting topic for many clinical applications. One limitation of VOI imaging that remains is the truncation artifact in the reconstructed 3-D volume. This artifact can either be a cupping effect towards the boundaries of the field-of-view (FOV) or an offset in the Hounsfield values of the reconstructed voxels. A new method for the correction of truncation artifacts in a collimated scan is introduced in this work. Scattered radiation still reaches the detector and is detected outside of the FOV, even if axial or lateral collimation is used. By reading out the complete detector area, we can use the scatter signal to estimate the truncated parts of the object: The scattered radiation outside the FOV is modeled as a convolution with a scatter kernel. This new approach is called Scatter Correction.

The reconstruction results using Scatter Convolution are at least as good or better than the results with a state-of-the-art method. Our results show that the use of scattered radiation outside the FOV improves image quality by $1.8 \%$.
\end{abstract}

\section{Introduction}

In many clinical applications, intra-procedural imaging is required. This is often done with fluoroscopy. Sometimes the information out of these 2-D images is not enough. Then a 3-D reconstruction is desirable, e.g. for a fluoroscopic overlay [1]. In order to reduce the patient dose, the X-rays are collimated so that only a small part of the detector size is used.

Collimated projection data leads to a problem when using filtered backprojection for 3-D reconstruction. Strong truncation artifacts appear at the boundary of the FOV, due to the ramp filtering of the projection data. The collimation introduces a sharp boundary into the projection data, which is amplified during the filtering step. These artificially high frequencies are backprojected into the volume and generate the truncation artifacts [2].

Due to the collimation, the scan does not cover the full spatial extent of the object. For an exact reconstruction, the full extent of the object has to be known 
from a prior scan [3], or a small part of the object has to be known [4]. If no prior knowledge is available, heuristic methods have to be applied. These methods are often called truncation correction in the literature. The better the heuristic fits the truncated object, the better the resulting reconstruction is. Some methods model the outside of the FOV, which was not measured [4,5]. Others require extrapolation only implicitly [6,7]. With respect to image quality, these heuristic methods provide acceptable results. In [8], an approach is presented that uses a filter to attenuate the radiation outside the FOV to a minimal dose. In a postprocessing step, this information is then used to solve the truncation problem. The approach presented here is similar to [8] as we also used a small amount of radiation outside the regular FOV. In contrast to [8], no additional dose has to be applied to the patient, as we employ the scattered radiation that is caused by the collimator edges and the object itself. This information is always present in a truncated FOV scenario but usually not measured.

Fig. 1 shows projection images of the skull of one patient. In Fig. 1(a), the full projection and in Fig. 1(b), the collimated projection are shown with the same grayscale window. Fig. 1(c) shows the same projection as in Fig. 1(b), but with a narrow windowing. It can be seen that the attenuated signal caused by the scattered radiation clearly depicts the shadow of the skull (cf. arrow). This information can be used to improve the image quality of the reconstruction inside the FOV.

\section{Materials and Methods}

In Fig. 2, an intensity profile through a collimated projection is plotted. The projection is divided into three parts: the FOV (area A), the area outside the FOV (area C), and the shadow of the collimator edge (area B).

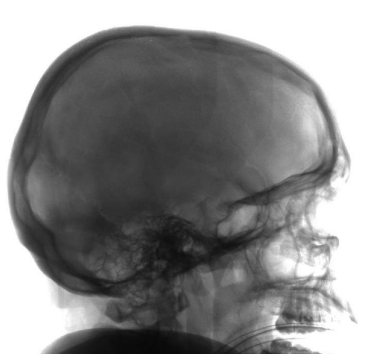

(a) Full projection, grayscale window $(400,2000)$.
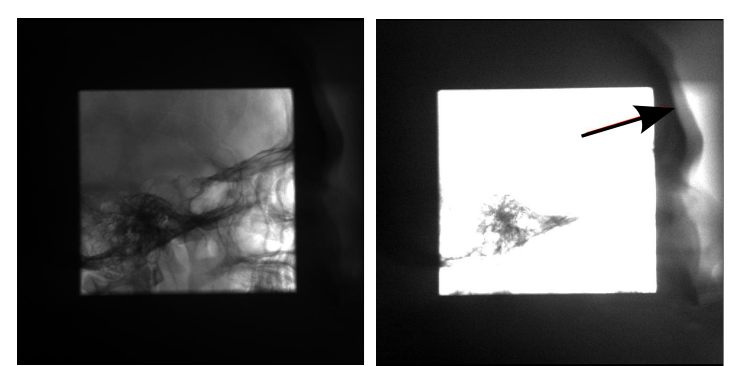

(b) Collimated projec- (c) Collimated projection, grayscale window tion, grayscale window $(400,2000)$.
$(400,620)$.

Fig. 1. Full and collimated projection images. 


\subsection{Edge Detection}

First, the edges of the collimator have to be detected. An edge is modeled as two lines that describe the beginning and the end of the collimator shadow (cf. Fig. 2). Since the truncation correction is applied in row direction, only the vertical collimator edges need to be detected.

For the initial detection, the Hough transformation (HT) is applied. After that, the collimator edge is approximated with a sigmoid curve in each row. On this sigmoid curve, the beginning and the end points of the collimator edge are calculated. RANSAC is used to fit continuous lines through the corresponding points of the four edges in each projection.

\subsection{Scatter Convolution}

A scatter model must be assumed before a correction is possible. There are different approaches for scatter estimation. They are either based on measurements, mathematical-physical models, or a combination of both ([9] gives an overview). One possibility is the beam-scatter-kernel superposition approach, a measurement-based method: The primary signal $\phi$ is convolved with a spatially invariant scatter kernel to estimate the scatter effect. $\phi$ is the primary signal without scatter and measured in the intensity domain $I / I_{0}$. Note that all the following calculations are done in the intensity domain instead of the line integral domain, because scatter is always additive to the intensity measured at the detector. The scatter estimation can be written as

$$
\phi_{\text {scatter }}=\phi * G \quad .
$$

$G$ is a scatter kernel and $\phi_{\text {scatter }}$ is the resulting scatter estimate.

Another measurement-based method presented in [10] is called collimatorshadow continuation method. This method is only possible if collimation is applied. The scattered radiation outside the FOV is measured. It is assumed that the scatter distribution in the FOV can be obtained by an interpolation between the measured boundary data. An example for this estimation is shown in Fig. 2.

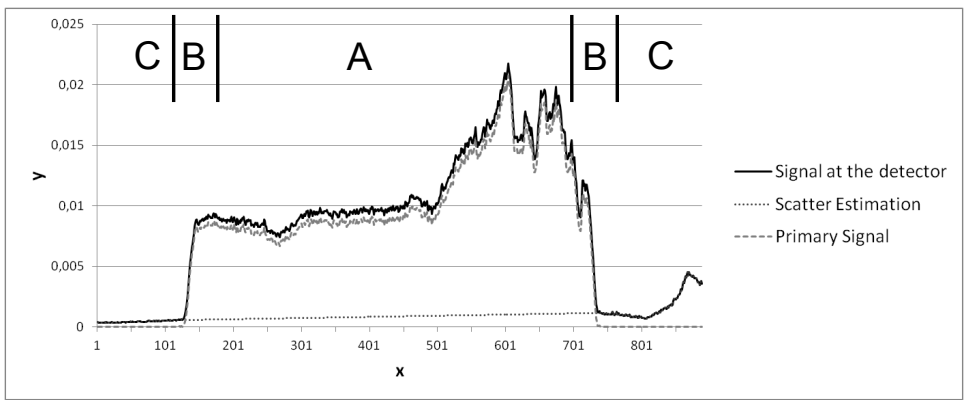

Fig. 2. Scatter estimation. 
The dotted line represents the scatter estimate. Inside the FOV (area A), this estimate is linearly interpolated using areas B and C. The signal at the detector is defined as

$$
\phi_{T}=\phi+S_{c}
$$

$\phi_{T}$ is the intensity measured at the detector. $\phi$ is the primary signal and $S_{c}$ is the scatter estimate using linear interpolation and measured data. Both scatter estimation models are used in the following.

Next, a suitable scatter correction method is needed. In [9], several are presented. For this approach, a projection-based deterministic scatter compensation approach is used. The initial equation is

$$
\phi_{T}=\phi+H(\phi) \quad .
$$

$H(\phi)=T \cdot(\phi * G)$ transforms the primary signal to the estimated scatter. By rearranging, the following fixed-point equation is found

$$
\phi=\phi_{T}-H(\phi) \quad .
$$

Let the fixed-point be $\phi=\phi_{c}$. If $\phi_{c}$ is the primary signal, then $H(\phi)$ is the correct scatter. After subtracting $H(\phi)$ from $\phi_{T}$, the primary signal is gained. Both $\phi$ and $H(\phi)$ are non-negative, since they represent intensities. Out of this equation, an iterative subtractive algorithm with relaxation can be derived [9]

$$
\phi^{(n+1)}=\phi^{(n)}+\lambda^{(n)}\left(\phi_{T}-\left(\phi^{(n)}+H\left(\phi^{(n)}\right)\right)\right) \quad .
$$

$\lambda$ is the step size of the iteration and $n$ the current iteration number. This equation is reformulated by inserting $\phi_{T}=\phi+S_{c}$, where $S_{c}$ is the initial scatter estimate from Eq. 2

$$
\begin{gathered}
\phi^{(n+1)}=\phi^{(n)}+\lambda^{(n)}\left(S_{c}-H\left(\phi^{(n)}\right)\right) \\
\text { with: } H\left(\phi^{(n)}\right)=T \cdot\left(\phi^{(n)} * G\right)
\end{gathered},
$$

$T$ is a scaling factor to adjust the estimated scatter to the initial scatter estimate $S_{c}$. $G$ is assumed to be a Gaussian kernel here. The results presented were calculated with two different Gaussians. The first kernel has a size of 150 pixels with $\sigma=10$, the second kernel has a size of 300 pixels and $\sigma=30$. Convolution is done row-wise.

$\phi^{(0)}=S_{c}-\phi_{T}$. Sometimes this value can be negative. To avoid this, only a small constant scatter is subtracted from the total signal. This constant is the minimum of the first and last pixel of the area $\mathrm{C}$ of the initial scatter estimate. Areas $\mathrm{B}$ and $\mathrm{C}$ of $\phi^{0}$ are extrapolated with the common water cylinder correction for the initial estimate.

Convolution is done in frequency domain. 40 iterations are performed with a constant step size of 0.1 . After each iteration, the area $\mathrm{A}$ of $\phi^{(n+1)}$ is replaced with the initial $\phi^{(0)}$. When a pixel in $\phi^{(n+1)}$ gets negative, the value from $\phi^{(n)}$ is taken. 
Table 1. Error measurements.

\begin{tabular}{clc}
\hline & CC & SSIM \\
\hline Dataset 1 Water cylinder correction & 0.9461 & 0.9444 \\
Scatter Convolution (kernel size 150, $\sigma=10$ ) & 0.9589 & 0.9527 \\
Scatter Convolution (kernel size 300, $\sigma=30$ ) & 0.9593 & 0.9533 \\
Dataset 2 Water cylinder correction & 0.9228 & 0.9224 \\
Scatter Convolution (kernel size 150, $\sigma=10$ ) & 0.9403 & 0.9368 \\
Scatter Convolution (kernel size 300, $\sigma=30$ ) & 0.9401 & 0.9367 \\
\hline
\end{tabular}

\section{Results}

We used two different datasets for evaluation. Both datasets were reconstructed using the new Scatter Correction approach and the water cylinder correction. Quantitative measurements use the correlation coefficient (CC) and the structural similarity (SSIM) index, since a reference dataset without collimation exists.

The results for dataset 1 and 2 are shown in Fig. 3 and 4 and Tab. 1. Scatter Convolution shows no cupping artifact. In the water cylinder correction, an intensity increase towards the boundary of the FOV is visible (cf arrows). At the boundary of the FOV, Scatter Correction retains more details than the water cylinder correction. Quantitatively, Scatter Correction also performs better than water cylinder correction.

\section{Discussion}

The method Scatter Convolution does not work when no scattered radiation is measured. This can however be detected, and only the normal water cylinder correction can be applied. Thus, the method performs at least as good as the water

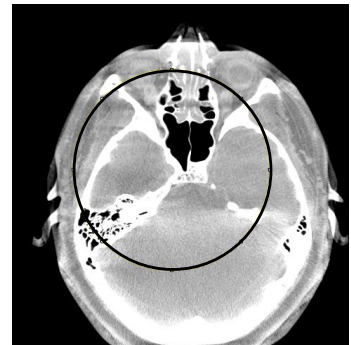

(a) Reference struction.

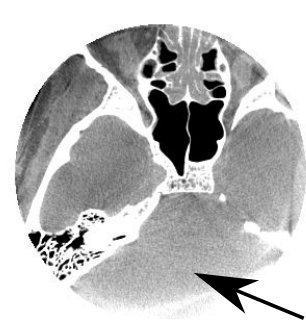

recon- (b) Water cylinder correction.

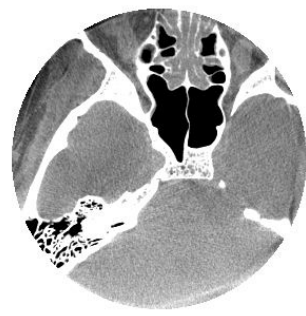

(c) Scatter Convolution approach.

Fig. 3. Results of dataset 1. Corrected reconstructions shown for FOV denoted by circle in reference.Grayscale window $(600,1400)$. 
cylinder correction. Further improvements could come from a refined physical model. Here, only simple scatter estimation and correction methods were used, which already lead to good reconstruction results.

\section{References}

1. Chintalapani G, Chinnadurai P, Maier A, Shaltoni H, Morsi H, Mawad M. The Value of Volume of Interest (VOI) C-arm CT Imaging in the Endovascular Treatment of Intracranial Aneurysms - A Feasibility Study. Procs ASNR. 2012;12-O1509-ASNR.

2. Zeng L. Medical Image Reconstruction. 1st ed. Heidelberg: Springer Verlag; 2010.

3. Kolditz D, Kyriakou Y, Kalender WA. Volume-of-interest (VOI) imaging in C-arm flat-detector CT for high image quality at reduced dose. Med Phys. 2010;37(6):2719-2730.

4. Kudo H, Courdurier M, Noo F, Defrise M. Tiny a priori knowledge solves the interior problem in computed tomography. Phys Med Biol. 2008;53(9):2207-2231.

5. Hsieh J, Chao E, Thibault J, Grekowicz B, Horst A, McOlash S. A novel algorithm to extend the CT scan field-of-view. Med Phys. 2004;31(9):2385-2391.

6. Dennerlein F, Maier A. Region-of-interest reconstruction on medical c-arms with the ATRACT algorithm. Medical Imaging 2012: Physics of Medical Imaging. 2012;8313:83131B-83131B-9.

7. Xia Y, Maier A, Dennerlein F, Hofmann HG, Hornegger J. Efficient 2D Filtering for Cone-Beam VOI Reconstruction. IEEE MIC. 2012;P. to appear.

8. Chityala R, Hoffmann KR, Bednarek DR, Rudin S. Region of Interest (ROI) Computed Tomography. Proc Soc Photo Opt Instrum Eng. 2004;5745(1):534-541.

9. Rührnschopf EP, Klingenbeck K. A general framework and review of scatter correction methods in x-ray cone-beam computerized tomography. Part 1+2. Med Phys. 2011;38(7):4296-4311, 5186-5199.

10. Siewerdsen JH, Daly MJ, Bakhtiar B, Moseley DJ, Richard S. A simple direct method for x-ray scatter estimation and correction in digital radiography and conebeam CT. Med Phys. 2006;33(1):187-197.
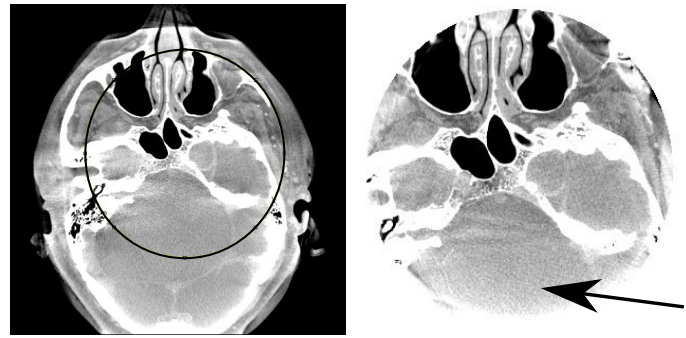

(a) Reference struction. recon- (b) Water cylinder correction.

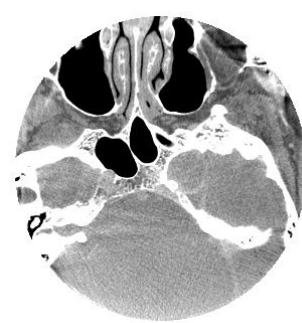

(c) Scatter Convolution approach.

Fig. 4. Results of dataset 2. Corrected reconstructions shown for FOV denoted by circle in reference. Grayscale window $(600,1400)$. 